Christian P. Robert • George Casella

\title{
Introducing Monte Carlo Methods with R
}

ei Springer 


\section{Contents}

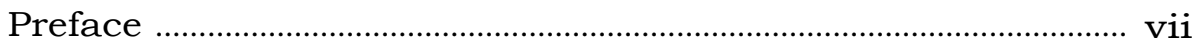

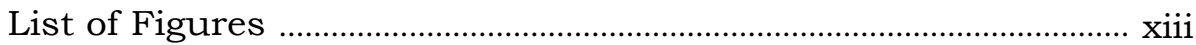

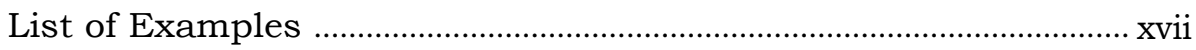

1 Basic R Programming ………………………………………….... 1

1.1 Introduction ……………………………………………………. 2

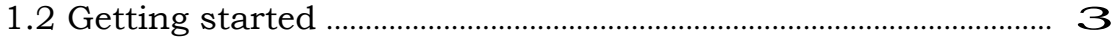

1.3 R objects ………………………………………………………………. 5

1.3.1 The vector class ........................................................................ 6

1.3.2 The matrix, array, and factor classes ………………………... 9

1.3.3 The list and data.frame classes ........................................... 12

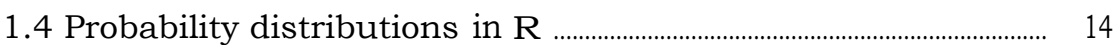

1.5 Basic and not-so-basic statistics ...................................................... 14

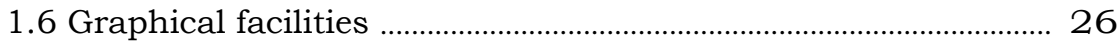

1.7 Writing new R functions ………………………………………………....

1.8 Input and output in $R \quad \underline{35}$

1.9 Administration of R objects ............................................................

1.10 The mosm package ……………………………………………………...... 36

1.11 Additional exercises .........................................................................................

2 Random Variable Generation ......................................................... 41

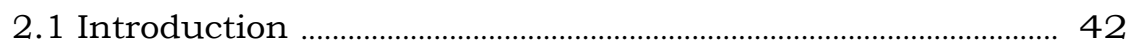

2.1.1 Uniform simulation ………………………………………... 42

2.1.2 The inverse transform …………………………………………... 44

2.2 General transformation methods ......................................................... 46

2.2.1 A normal generator ………………………………………….... 47

2.2.2 Discrete distributions ............................................................... 48

2.2.3 Mixture representations ……………………………………..... 50

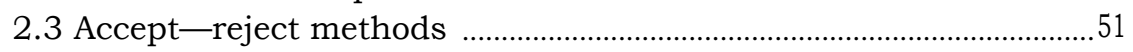


2.4 Additional exercises

3 Monte Carlo Integration ............................................................ 61

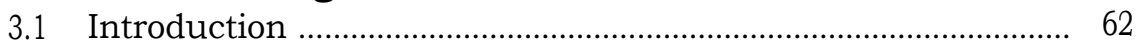

3.2 Classical Monte Carlo integration …........................................... 65

3.3 Importance sampling .................................................................. 69

3.3.1 An arbitrary change of reference measure ...................... 69

3.3.2 Sampling importance resampling .................................. $\quad 75$

3.3.3 Selection of the importance function ............................. 78

3.4 Additional exercises .................................................................. 86

4 Controlling and Accelerating Convergence .................................. 89

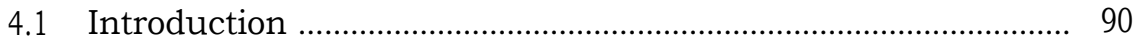

4.2 Monitoring variation ....................................................................... 91

4.3 Asymptotic variance of importance sampling estimators ......... 92

4.4 Effective sample size and perplexity ............................................ 98

4.5 Simultaneous monitoring …..................................................... 100

4.6 Rao-Blackwellization and deconditioning ............................... 107

4.7 Acceleration methods ............................................................. 111

4.7.1 Correlated simulations ...................................................... 111

4.7.2 Antithetic variables ............................................................. 113

4.7.3 Control variates ............................................................... 116

4.8 Additional exercises ................................................................. 122

$5 \quad$ Monte Carlo Optimization ........................................................... 125

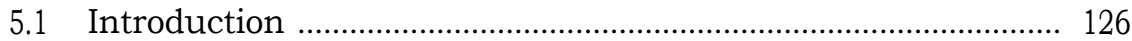

5.2 Numerical optimization methods .............................................. 127

5.3 Stochastic search ..................................................................... 130

5.3.1 A basic solution ................................................................ 130

5.3.2 Stochastic gradient methods ........................................... 136

5.3.3 Simulated annealing .......................................................... 140

5.4 Stochastic approximation ............................................................ 146

5.4.1 Optimizing Monte Carlo approximations ........................ 146

5.4.2 Missing-data models and demarginalization .................... 150

5.4.3 The EM algorithm ........................................................... 152

5.4.4 Monte Carlo EM ................................................................ 157

5.5 Additional exercises ................................................................ 163

6 Metropolis-Hastings Algorithms ..................................................... 167

6.1 Introduction ...................................................................... 168

6.2 A peek at Markov chain theory ..................................................... 168

6.3 Basic Metropolis-Hastings algorithms ......................................... 170

6.3.1 A generic Markov chain Monte Carlo algorithm ........... 171

6.3.2 The independent Metropolis-Hastings algorithm .......... 175

6.4 A selection of candidates ............................................................ 182 
6.4.1 Random walks

6.4.2 Alternative candidates ................................................................ 185

6.5 Acceptance raten

6.6 Additional exercises

7 Gibbs Samplers

7.1 Introduction

200

7.2 The two-stage Gibbs sampler

200

7.3 The multistage Gibbs sampler

206

7.4 Missing data and latent variables $\quad \underline{209}$

7.5 Hierarchical structures ................................................................ 221

7.6 Ot her considerations ........................................................................ 224

7.6.1 Reparameterization ................................................................. 224

7.6.2 Rao-Blackwellization ........................................................... 227

7.6.3 Ietropolis within Gibbs and hybrid strategies 230

7.6.4 Improper priors $\quad 232$

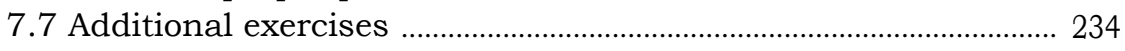

8 Monitoring and Adaptation for MCMC Algorithms 237

8.1 Introduction ............................................................................ 238

8.2 Monitoring what and why 238

8.2.1 Convergence to the st ationary dist ribution 238

8.2.2 Convergence of averages ....................................................... 240

8.2.3 Approximating iid sampling ……....................................... 240

8.2.4 The coda package ..................................................................... 241

8.3 NIonitoring convergence to stationarity .......................................... 242

8.3.1 Graphical diagnoses ........................................................... 242

8.3.2 Nonparametric tests of stationarity ....................................... 243

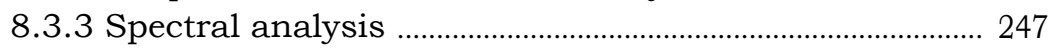

8.4 Monitoring convergence of averages .............................................. 250

8.4.1 Graphical diagnoses $\quad 250$

8.4.2 Within and between variances $\quad \underline{253}$

8.4.3 Effective sample size $\quad \underline{255}$

8.4.4 Fixed-width batch ineans ................................................... 257

8.5 Adaptive MCMC 258

8.5.1 Cautions about adaptation 258

8.5.2 The amcmc package .............................................................. 264

8.6 Additional exercises ......................................................................... 267

References .................................................................................................................. 269

Index of $\mathbf{R}$ Terms

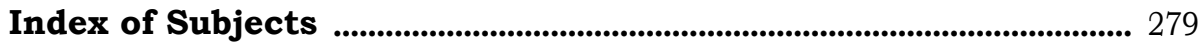

